\title{
Commutators of Square Functions Related to Fractional Differentiation for Second-Order Elliptic Operators
}

\author{
Xiongtao Wu, ${ }^{1}$ Wenyu Tao, ${ }^{2}$ Yanping Chen $\mathbb{D}^{3},{ }^{3}$ and Kai Zhu $\mathbb{D}^{4}$ \\ ${ }^{1}$ Department of Mathematics, School of Mathematics and Statistics, Hengyang Normal University, Hengyang 421008, China \\ ${ }^{2}$ School of Mathematics and Physics, University of Science and Technology Beijing, Beijing 100083, China \\ ${ }^{3}$ Department of Applied Mathematics, School of Mathematics and Physics, University of Science and Technology Beijing, \\ Beijing 100083, China \\ ${ }^{4}$ School of Mathematical Sciences, Beijing Normal University, Beijing 100875, China \\ Correspondence should be addressed to Yanping Chen; yanpingch@126.com
}

Received 11 June 2018; Accepted 7 November 2018; Published 2 December 2018

Academic Editor: Yoshihiro Sawano

Copyright (C) 2018 Xiongtao Wu et al. This is an open access article distributed under the Creative Commons Attribution License, which permits unrestricted use, distribution, and reproduction in any medium, provided the original work is properly cited.

Let $L=-\operatorname{div}(A \nabla)$ be a second-order divergence form elliptic operator, where $A$ is an accretive $n \times n$ matrix with bounded measurable complex coefficients in $\mathbb{R}^{n}$. In this paper, we mainly establish the $L^{p}$ boundedness for the commutators generated by $b \in I_{\alpha}(B M O)$ and the square function related to fractional differentiation for second-order elliptic operators.

\section{Introduction}

Let $T$ be a linear operator in a measurable function space; then, the commutator formed by $T$ and $b \in L_{\mathrm{loc}}\left(\mathbb{R}^{n}\right)$ is defined by

$$
[b, T] f(x):=b(x) T f(x)-T(b f)(x) .
$$

For $b \in L_{\text {loc }}\left(\mathbb{R}^{n}\right)$, set

$$
M(b, Q)=\frac{1}{|Q|} \int_{Q}\left|b(y)-b_{\mathrm{Q}}\right| d y,
$$

where $Q$ is a cube in $\mathbb{R}^{n}$ and $b_{Q}=|Q|^{-1} \int_{Q} b(y) d y$. Then, the $B M O$ space is defined as

$$
\begin{aligned}
& \text { BMO } \\
& =\left\{b \in L_{\mathrm{loc}}\left(\mathbb{R}^{n}\right):\|b\|_{*}:=\sup _{{\mathrm{Q} \subset \mathbb{R}^{n}}} M(b, Q)<\infty\right\} .
\end{aligned}
$$

Let $0<\alpha<1$, and consider the fractional differentiation operators of even and odd parities, defined for tempered distributions $f \in S^{\prime}\left(\mathbb{R}^{n}\right)$, by $\widehat{D^{\alpha} f}(\xi)=|\xi|^{\alpha} \widehat{f}(\xi)$. Let $I_{\alpha}$ be the Riesz potential operator of order $\alpha$ and be defined in the space of tempered distribution modulo polynomials by setting $\widehat{I_{\alpha} f}(\xi)=|\xi|^{-\alpha} \widehat{f}(\xi)$. The BMO Sobolev space $I_{\alpha}(B M O)$ is the image of $B M O$ under $I_{\alpha}$. Equivalently, $b \in I_{\alpha}(B M O)$ if and only if $D^{\alpha} b \in B M O$. Let $b \in \operatorname{Lip}_{\alpha}$, subsequently, if $b$ satisfies $\|b\|_{\operatorname{Lip}_{\alpha}}=\sup |b(x)-b(y)| /|x-y|^{\alpha}<\infty$, in which the supremum is taken over all $x, y \in \mathbb{R}^{n}$ and $x \neq y$. For $\alpha \in(0,1), I_{\alpha}(B M O)$ is a space of functions modulo constants that is properly contained in $\operatorname{Lip}_{\alpha}$ (see $[1,2]$ ).

Before presenting our main theorem, we introduce the second-order elliptic operator $L$ as follows: For $\xi=$ $\left(\xi_{1}, \ldots, \xi_{n}\right) \in \mathbb{C}^{n}$, denote its complex conjugate $\left(\bar{\xi}_{1}, \ldots, \bar{\xi}_{n}\right)$ by $\bar{\xi}$. Let $A=A(x)$ be an $n \times n$ matrix of complex $L^{\infty}$ coefficients defined on $\mathbb{R}^{n}$ that satisfy the ellipticity condition:

$$
\begin{aligned}
\lambda|\xi|^{2} & \leq \operatorname{Re} A \xi \cdot \bar{\xi} \\
|A \xi \cdot \bar{\zeta}| & \leq \Lambda|\xi||\zeta|,
\end{aligned}
$$

for $\xi, \zeta \in \mathbb{C}^{n}$ and for some $\lambda, \Lambda$ such that $0<\lambda \leq \Lambda<\infty$. Here, the inner product notation $u \cdot v=u_{1} v_{1}+\cdots+u_{n} v_{n}$. Therefore, $A \xi \cdot \bar{\zeta} \equiv \sum_{j, k} a_{j, k}(x) \xi_{k} \bar{\zeta}_{j}$. Associated with such a matrix $A$, we define a second-order divergence form operator:

$$
L f=-\operatorname{div}(A \nabla f)=-\sum_{j=1}^{n} \partial_{j}\left((A \nabla f)_{j}\right),
$$


which we interpret in the usual weak sense via a sesquilinear form. The operator $-L$ generates a semigroup $\left(e^{-t L}\right)_{t>0}$ and the gradient of the semigroup $\left(\nabla t^{1 / 2} e^{-t L}\right)_{t>0}$.

In this paper, we first present a general criterion for weak-type $(p, p)$ boundedness of commutators with square functions and $b \in I_{\alpha}(B M O)$.

Theorem 1. Let $1<p<2,0<\alpha \leq 1, b \in I_{\alpha}(B M O)$, and $m$ be an integer greater than 1 . Let $E$ and $F$ be two closed subsets of $\mathbb{R}^{n}$ with a Euclidean distance $d(E, F)$ between each other, and let $\left\{T_{s}\right\}_{s>0}$ be a family of sublinear operators acting on $L^{2}\left(\mathbb{R}^{n}\right)$. Assume that, for $t>0$ and $2<p_{1}<\infty$,

$$
\begin{gathered}
\left\|\left(\int_{0}^{\infty}\left|\left[b, T_{s}\left(\mathscr{I}-e^{-t L}\right)^{m}\right] f\right|^{2} \frac{d s}{s}\right)^{1 / 2}\right\|_{L^{p_{1}}(F)} \\
\leq C t^{\eta}\left(\frac{d(E, F)^{2}}{t}\right)^{-(m-\eta)}\|f\|_{L^{p}(E)},
\end{gathered}
$$

where $\eta=(1 / 2)\left(n / p_{1}-n / p\right)$. Furthermore, if

$$
\begin{gathered}
\left\|\left(\int_{0}^{\infty}\left|T_{s} f\right|^{2} \frac{d s}{s}\right)^{1 / 2}\right\|_{L^{2}} \leq C\left\|D^{\alpha} f\right\|_{L^{2}}, \\
\left\|\left(\int_{0}^{\infty}\left|\left[b, T_{s}\right] f\right|^{2} \frac{d s}{s}\right)^{1 / 2}\right\|_{L^{2}} \leq C\|f\|_{L^{2}} .
\end{gathered}
$$

Then, we have

$$
\begin{aligned}
& \left|\left\{x \in \mathbb{R}^{n}:\left|\left(\int_{0}^{\infty}\left|\left[b, T_{s}\right] f(x)\right|^{2} \frac{d s}{s}\right)^{1 / 2}\right|>\lambda\right\}\right| \\
& \leq C \lambda^{-p}\|f\|_{L^{p}}^{p} .
\end{aligned}
$$

We recall a square function, which is representative of larger classes of square functions associated with $L$, given as follows [3]:

$$
g_{L} f(x)=\left(\int_{0}^{\infty}\left|t^{1 / 2} \nabla e^{-t L} f(x)\right|^{2} \frac{d t}{t}\right)^{1 / 2} .
$$

In 2007, Aushcer [3] proved that $\left\|g_{L} f\right\|_{L^{p}} \sim\|f\|_{L^{p}}$ for $q_{L}<$ $p<\widetilde{q}_{L}$. The interval $\left(q_{L}, \widetilde{q}_{L}\right)$ is the maximal open interval required for the semigroup $\left(\sqrt{L} \nabla e^{-t L}\right)_{t>0}$ to be $L^{p}$ bounded. We recall that $\left(p_{L}, \widetilde{p}_{L}\right)$ is the maximal open interval required for the semigroup $\left(e^{-t L}\right)_{t>0}$ to be $L^{p}$ bounded. In [3], the author has shown in general that $p_{L}=1, \widetilde{p}_{L}=\infty$ if $n=1,2$; $p_{L}<2 n /(n+2), \tilde{p}_{L}>2 n /(n-2)$ if $n \geq 3$ and $p_{L}=q_{L}$.

Many researchers have contributed to the commutators associated with the second-order elliptic operator, and among the numerous studies, some related to development and applications have been cited herein [4-9]. In particular, commutators with fractional differentiations associated with $L$ play an important role in the theory of linear partial differential equations and harmonic analysis [10-14]. Naturally, the case of the commutators of square functions being related to fractional differentials associated with $L$ is worth studying.
In this paper, we define a square function related to the fractional differential operator associated with $L$ as follows:

$$
G_{L}^{\alpha} f(x)=\left(\int_{0}^{\infty}\left|t^{1 / 2} \nabla e^{-t L} f(x)\right|^{2} \frac{d t}{t^{1+\alpha}}\right)^{1 / 2},
$$

$$
0<\alpha<1 \text {. }
$$

Moreover, for $0<\alpha<1$ and $b \in I_{\alpha}(B M O)$, the commutator of $G_{L}^{\alpha}$ can be defined by

$$
G_{L ; b}^{\alpha} f(x)=\left(\int_{0}^{\infty}\left|\left[b, t^{1 / 2} \nabla e^{-t L}\right] f(x)\right|^{2} \frac{d t}{t^{1+\alpha}}\right)^{1 / 2} .
$$

In this paper, we also establish the $L^{p}$ boundedness for $G_{L ; b}^{\alpha}$.

Theorem 2. Let $L$ be a second-order elliptic operator in divergence form defined by (5), $0<\alpha<1$, and $b \in I_{\alpha}(B M O)$. Then, for $p_{L}<p \leq 2$, we have

$$
\left\|G_{L ; b}^{\alpha} f\right\|_{L^{p}} \leq C\left\|D^{\alpha} b\right\|_{B M O}\|f\|_{L^{p}} .
$$

The remainder of this paper is organised as follows: in Section 2, we present some lemmas that play an important role in the proof of the main results; in Section 3, we prove Theorem 1; in Section 4, we prove Theorem 2. For $p \geq 1$, $p^{\prime}$ denotes the dual exponent of $p$, i.e., $p^{\prime}=p /(p-1)$. Throughout this paper, the letter " $C$ " will stand for a positive constant that is independent of the essential variables but will not necessarily have the same value for each occurrence.

\section{Preliminary Lemmas}

The second-order elliptic operator $L$ in divergence form is defined by (5) and has the following off-diagonal estimates (see $[3,4,9]$ and references therein).

Lemma 3 (see $[3,4,9]$ ). Let $L$ be a second-order elliptic operator defined by (5), let $E$ and $F$ be two closed sets of $\mathbb{R}^{n}$, and let set $d(E, F)$ denote the distance between $E$ and $F$. Then, for $t>0$, the complex-valued function $f$, and vector-valued functions $\vec{f}$, the following statements hold:

(i) Let $q_{L}<p \leq q<\widetilde{q}_{L}$ and $f \in L^{p}\left(\mathbb{R}^{n}\right)$ supported in $E$,

$$
\begin{aligned}
& \left\|t^{1 / 2} \nabla e^{-t L} f\right\|_{L^{q}(F)} \\
& \quad \leq C t^{-(n / 2)(1 / p-1 / q)} e^{-c\left(d(E, F)^{2} / t\right)}\|f\|_{L^{p}(E)} ;
\end{aligned}
$$

(ii) Let $p_{L}<p \leq q<\widetilde{p}_{L}, f \in L^{p}\left(\mathbb{R}^{n}\right)$ supported in $E$ and $k \in \mathbb{N}$,

$$
\begin{aligned}
& \left\|(t L)^{k} e^{-t L} f\right\|_{L^{q}(F)} \\
& \quad \leq C t^{-(n / 2)(1 / p-1 / q)} e^{-c\left(d(E, F)^{2} / t\right)}\|f\|_{L^{p}(E)} ;
\end{aligned}
$$

(iii) Let $p_{L}<p \leq q<\tilde{p}_{L}$ and $\vec{f} \in \dot{L}_{1}^{p}\left(\mathbb{R}^{n}\right)$ supported in $E$,

$$
\begin{aligned}
& \left\|t \nabla e^{-t L} \operatorname{div} \vec{f}\right\|_{L^{q}(F)} \\
& \quad \leq C t^{-(n / 2)(1 / p-1 / q)} e^{-c\left(d(E, F)^{2} / t\right)}\|\vec{f}\|_{L^{p}(E)} .
\end{aligned}
$$


In particular, if we choose $E=F=\mathbb{R}^{n}$, the abovementioned $L^{p}-L^{q}$ off-diagonal estimates become $L^{p}-L^{q}$ estimates.

Another very useful and well-known lemma for offdiagonal estimates is introduced here, which could be proved by using a similar argument for the proof of a previous lemma [9, lemma 2.3].

Lemma 4 ([3,9]). Let $1<p \leq q<\infty$ and $E, F$ be arbitrary closed subsets of $\mathbb{R}^{n}$. Assume that the two families of the operators $\left\{A_{t}\right\}_{t>0}$ and $\left\{B_{s}\right\}_{s>0}$ satisfy the following offdiagonal estimates:

$$
\begin{aligned}
& \left\|A_{t}\left(g \chi_{E}\right)\right\|_{L^{q}(F)} \leq C e^{-d(E, F)^{2} / c t}\|g\|_{L^{q}(E)}, \\
& \left\|B_{s}\left(g \chi_{E}\right)\right\|_{L^{q}(F)} \leq C s^{(n / 2)(1 / q-1 / p)} e^{-d(E, F)^{2} / c s}\|g\|_{L^{p}(E)} .
\end{aligned}
$$

Then, for $t, s>0$ and $f \in L^{p}\left(\mathbb{R}^{n}\right)$ supported in $E$, we have

$$
\begin{aligned}
& \left\|A_{t} B_{s} f\right\|_{L^{q}(F)} \\
& \quad \leq C s^{(n / 2)(1 / q-1 / p)} e^{-d(E, F)^{2} / c \max \{t, s\}}\|f\|_{L^{p}(E)} .
\end{aligned}
$$

Next, let us introduce a criterion that deals with the boundedness of the commutators of the operators satisfying $L^{p} \longrightarrow L^{q}$ off-diagonal estimates, which can be proved in [15].

Lemma 5 (see [15]). Let $E$ and $F$ be two closed subsets of $\mathbb{R}^{n}$ with a Euclidean distance $d(E, F)$, and let $\left\{T_{s}\right\}_{s>0}$ be a family of sublinear operators acting on $L^{2}\left(\mathbb{R}^{n}\right)$. Assume that, for $1 \leq$ $p \leq q \leq \infty$ and $f \in C_{0}^{\infty}\left(\mathbb{R}^{n}\right)$ with supp $f \subset E$,

$$
\left\|T_{s} f\right\|_{L^{q}(E)} \leq C s^{(1 / 2)(n / q-n / p)} e^{-d(E, F)^{2} / c s}\|f\|_{L^{p}(E)} .
$$

If $\max \{\operatorname{diam} E, \operatorname{diam} F\} \leq C d(E, F)$, then for $0<\alpha<1$ and $b \in \operatorname{Lip}_{\alpha}\left(\mathbb{R}^{n}\right)$, we have

$$
\begin{aligned}
& \left\|\left[b, s^{-\alpha / 2} T_{s}\right] f\right\|_{L^{q}(F)} \\
& \quad \leq C s^{(1 / 2)(n / q-n / p)} e^{-d(E, F)^{2} / c s}\|b\|_{\operatorname{Lip}_{\alpha}}\|f\|_{L^{p}(E)},
\end{aligned}
$$

where $C$ is independent of $s, b$, and $f$.

The following two lemmas are about the $L^{p}-L^{q}$ offdiagonal estimates related to some commutators of the Lipschitz function and semigroups for second-order elliptic operators.

Lemma 6 (see [16]). Let $E$ and $F$ be two closed subsets of $\mathbb{R}^{n}$ with a Euclidean distance $d(E, F)$. Assume that $0<\alpha<1$ and $b \in \operatorname{Lip}_{\alpha}\left(\mathbb{R}^{n}\right)$ and $0 \leq \gamma \leq 1$. If $\max \{\operatorname{diamE}, \operatorname{diamF}\} \leq$ $C d(E, F)$, then for $s>0$ and $f \in L^{p}\left(\mathbb{R}^{n}\right)$ supported in $E$, we obtain the following for some $\varepsilon>0$ :

$$
\begin{aligned}
& \left\|s^{-\alpha / 2}\left[b, s^{\gamma / 2} L^{\gamma / 2} e^{-s L}\right] f\right\|_{L^{q}(F)} \\
& \leq C s^{(1 / 2)(n / q-n / p)} e^{-d(E, F)^{2} / c s}\|b\|_{L i p_{\alpha}}\|f\|_{L^{p}(E)}, \\
& p_{L}<p \leq q<2+\varepsilon .
\end{aligned}
$$

Lemma 7 (see [16]). Let $L$ be the second-order elliptic operator in divergence form defined by (5), E and $F$ be two closed sets of $\mathbb{R}^{n}$, and $d(E, F)$ express the distance between $E$ and $F$. Assume that $0<\alpha<1, b \in \operatorname{Lip}_{\alpha}\left(\mathbb{R}^{n}\right)$, and $0 \leq \gamma \leq 1$. Then, for $s>0$, $f \in L^{p}\left(\mathbb{R}^{n}\right)$ supported in $E$, we obtain

$$
\begin{aligned}
& \left\|\left[b, s^{(1-\alpha) / 2} \nabla e^{-t L}\right] f\right\|_{L^{q}(F)} \\
& \leq C\|b\|_{L i p_{\alpha}} s^{-(n / 2)(1 / p-1 / q)} e^{-d(E, F)^{2} / c s}\|f\|_{L^{p}(E)}, \\
& p_{L}<p \leq q<\widetilde{p}_{L},
\end{aligned}
$$

where $C$ is independent of $s, b, f$.

\section{Proof of Theorem 1}

For any fixed $\lambda>0$, without loss of generality, we may assume that $f \in L^{p}\left(\mathbb{R}^{n}\right)$ is nonnegative. Let us write $M$ for the Hardy-Littlewood maximal function. We use the CalderónZygmund decomposition for $f(x)^{p}$ at height $\lambda^{p}$. Then, there exists a collection of pairwise disjoint cubes $\left\{Q_{j}\right\}_{j}$ such that

$$
\left\{x \in \mathbb{R}^{n}: M\left(f^{p}\right)(x)^{1 / p}>\lambda\right\}=\bigcup_{j} Q_{j}
$$

and they satisfy the following property:

$$
\lambda \leq\left(\frac{1}{\left|Q_{j}\right|} \int_{Q_{j}} f(x)^{p} d x\right)^{1 / p} \leq C \lambda .
$$

Then, we write $f=g+h=g+\sum_{j} h_{j}$, where

$$
\begin{aligned}
g(x)= & f(x) \chi_{\mathbb{R}^{n} \backslash \bigcup_{j} Q_{j}} \\
& +\sum_{j}\left(\frac{1}{\left|Q_{j}\right|} \int_{Q_{j}} f(y) d y\right) \chi_{Q_{j}}(x), \\
h_{j}(x)= & \left(f(x)-\frac{1}{\left|Q_{j}\right|} \int_{Q_{j}} f(y) d y\right) \chi_{Q_{j}}(x) .
\end{aligned}
$$

After estimating (24), $p>1$ and the standard arguments yield $0 \leq g(x) \leq C \lambda$ for almost every $x \in \mathbb{R}^{n}$. Then,

$$
\int_{Q_{j}} h_{j}(x) d x=0,
$$

and

$$
\left(\frac{1}{\left|Q_{j}\right|} \int_{Q_{j}}\left|h_{j}(x)\right|^{p} d x\right)^{1 / p} \leq C \lambda,
$$

hence

$$
\left|\left\{x \in \mathbb{R}^{n}:\left|\left(\int_{0}^{\infty}\left|\left[b, T_{s}\right] f(x)\right|^{2} \frac{d s}{s}\right)^{1 / 2}\right|>3 \lambda\right\}\right|
$$




$$
\begin{aligned}
& \leq\left|\left\{x \in \mathbb{R}^{n}:\left|\left(\int_{0}^{\infty}\left|\left[b, T_{s}\right] g(x)\right|^{2} \frac{d s}{s}\right)^{1 / 2}\right|>\lambda\right\}\right| \\
& +\left|\left\{x \in \mathbb{R}^{n}:\left|\left(\int_{0}^{\infty}\left|\left[b, T_{s}\right] h(x)\right|^{2} \frac{d s}{s}\right)^{1 / 2}\right|>2 \lambda\right\}\right| \\
& :=I+I I .
\end{aligned}
$$

We estimate every term separately. For $I$, we use (8) and the properties of $g$ to obtain

$$
\begin{aligned}
I & \leq \frac{1}{\lambda^{2}} \int_{\mathbb{R}^{n}} \int_{0}^{\infty}\left|\left[b, T_{s}\right] g(x)\right|^{2} \frac{d s}{s} d x \\
& \leq \frac{C}{\lambda^{2}} \int_{\mathbb{R}^{n}}|g(x)|^{2} d x \leq \frac{C \lambda^{2-p}}{\lambda^{2}} \int_{\mathbb{R}^{n}}|g(x)|^{p} d x \\
& \leq C \lambda^{-p}\|f\|_{L^{p}}^{p} .
\end{aligned}
$$

Now, we proceed with $I I$. Let us fix an integer $m \geq 1$. We write $t_{j}=\ell\left(Q_{j}\right)^{2}$, where $\ell\left(Q_{j}\right)$ stands for the side length of the cube $Q_{j}$. We use the notation $Q_{j}^{*}=2 Q_{j}$, where, in general, we write $\rho Q$ for the $\rho$-dilated $Q$, i.e., for the cube with the same centre as $Q$ and with the side length $\rho \ell(Q)$. Let $E^{*}=\mathbb{R}^{n} \backslash \bigcup_{j} Q_{j}^{*}$. Because

$$
\begin{aligned}
& \left(\int_{0}^{\infty}\left|\left[b, T_{s}\right] h(x)\right|^{2} \frac{d s}{s}\right)^{1 / 2} \\
& \quad=\left(\int_{0}^{\infty}\left|\sum_{j}\left[b, T_{s}\right] h_{j}(x)\right|^{2} \frac{d s}{s}\right)^{1 / 2} \\
& \quad \leq\left(\int_{0}^{\infty}\left|\sum_{j}\left[b, T_{s}\left(\mathscr{I}-e^{-t_{j} L}\right)^{m}\right] h_{j}(x)\right|^{2} \frac{d s}{s}\right)^{1 / 2} \\
& \quad+\left(\int_{0}^{\infty}\left|\sum_{j}\left[b, T_{s}\left(\mathscr{I}-\left(\mathscr{I}-e^{-t_{j} L}\right)^{m}\right)\right] h_{j}(x)\right|^{2} \frac{d s}{s}\right)^{1 / 2},
\end{aligned}
$$

we obtain

$$
\begin{aligned}
& I I \leq \mid\{x: \\
& \left.\left(\int_{0}^{\infty}\left|\sum_{j}\left[b, T_{s}\left(\mathscr{I}-e^{-t_{j} L}\right)^{m}\right] h_{j}(x)\right|^{2} \frac{d s}{s}\right)^{1 / 2}>\lambda\right\} \mid \\
& +\mid\{x: \\
& \left(\int_{0}^{\infty}\left|\sum_{j}\left[b, T_{s}\left(\mathscr{I}-\left(\mathscr{I}-e^{-t_{j} L}\right)^{m}\right)\right] h_{j}(x)\right|^{2} \frac{d s}{s}\right)^{1 / 2} \\
& >\lambda\}|\leq| \bigcup_{j} Q_{j}^{*}|+|\left\{x \in E^{*}:\right. \\
& \left.\left(\int_{0}^{\infty}\left|\sum_{j}\left[b, T_{s}\left(\mathscr{I}-e^{-t_{j} L}\right)^{m}\right] h_{j}(x)\right|^{2} \frac{d s}{s}\right)^{1 / 2}>\lambda\right\} \mid \\
& +\mid\{x: \\
& \left(\int_{0}^{\infty}\left|\sum_{j}\left[b, T_{s}\left(\mathscr{I}-\left(\mathscr{I}-e^{-t_{j} L}\right)^{m}\right)\right] h_{j}(x)\right|^{2} \frac{d s}{s}\right)^{1 / 2} \\
& >\lambda\} \mid:=I I_{1}+I I_{2}+I I_{3} .
\end{aligned}
$$

The first term can be estimated as follows:

$$
I I_{1} \leq \sum_{j}\left|Q_{j}^{*}\right| \leq \frac{C}{\lambda^{p}}\|f\|_{L^{p}}^{p} .
$$

Now, we complete the estimate of $I_{2}$. By Chebychev's inequality, we obtain

$$
\begin{aligned}
I I_{2} & \leq \frac{1}{\lambda^{p_{1}}}\left\|\left(\int_{0}^{\infty}\left|\sum_{j}\left[b, T_{s}\left(\mathscr{I}-e^{-t_{j} L}\right)^{m}\right] h_{j}(x)\right|^{2} \frac{d s}{s}\right)^{1 / 2}\right\|_{L^{p_{1}\left(E^{*}\right)}}^{p_{1}} \\
& =\left.\left.\frac{1}{\lambda^{p_{1}}}\left|\sup _{v} \int_{\mathbb{R}^{n}} \int_{0}^{\infty}\right| \sum_{j}\left[b, T_{s}\left(\mathscr{I}-e^{-t_{j} L}\right)^{m}\right] h_{j}(x)\right|^{2} \frac{d s}{s} v(x) d x\right|^{p_{1} / 2}
\end{aligned}
$$

where the supremum is taken over all the functions $v \in$ $L^{\left(p_{1} / 2\right)^{\prime}}\left(E^{*}\right)$ with $\|v\|_{L^{\left(p_{1} / 2\right)^{\prime}}\left(E^{*}\right)}=1$. We set

$$
S_{l}\left(Q_{j}\right)=2^{l+1} Q_{j} \backslash 2^{l} Q_{j},
$$

$$
S_{0}\left(Q_{j}\right)=2 Q_{j}
$$


Let us recall that $E^{*}=\mathbb{R}^{n} \backslash \bigcup_{j} Q_{j}^{*}$. Because supp $v \subset\left(2 Q_{j}\right)^{c}$, we have

$$
\begin{aligned}
& \left.\left.\left|\int_{\mathbb{R}^{n}} \int_{0}^{\infty}\right| \sum_{j}\left[b, T_{s}\left(\mathscr{I}-e^{-t_{j} L}\right)^{m}\right] h_{j}(x)\right|^{2} \frac{d s}{s} v(x) d x\right|^{1 / 2} \leq\left.\left.\left|\int_{\mathbb{R}^{n}} \int_{0}^{\infty}\right| \sum_{j}\left[b, T_{s}\left(\mathscr{I}-e^{-t_{j} L}\right)^{m}\right] h_{j}(x) v(x)^{1 / 2}\right|^{2} \frac{d s}{s} d x\right|^{1 / 2} \\
& \quad=\sum_{j}\left(\sum_{l=1}^{\infty} \int_{S_{l}\left(Q_{j}\right)} \int_{0}^{\infty}\left|\left[b, T_{s}\left(\mathscr{I}-e^{-t_{j} L}\right)^{m}\right] h_{j}(x) v(x)^{1 / 2}\right|^{2} \frac{d s}{s} d x\right)^{1 / 2} \\
& \quad \leq C \sum_{j}\left(\sum_{l=1}^{\infty}\left\|\left(\int_{0}^{\infty}\left|\left[b, T_{s}\left(\mathscr{I}-e^{-t_{j} L}\right)^{m}\right] h_{j}\right|^{2} \frac{d s}{s}\right)^{1 / 2}\right\|_{L^{p_{1}\left(S_{l}\left(Q_{j}\right)\right)}}^{2}\|v\|_{L^{\left(p_{1} / 2\right)^{\prime}}\left(S_{l}\left(Q_{j}\right)\right)}\right)^{1 / 2} \\
& \quad \leq C \sum_{j}\left(\sum_{l=1}^{\infty} t_{j}^{2 \eta}\left(\frac{d\left(S_{l}\left(Q_{j}\right), Q_{j}\right)^{2}}{t_{j}}\right)^{-2(m-\eta)}\left\|h_{j}\right\|_{L^{p}\left(Q_{j}\right)}^{2}\|v\|_{L^{\left(p_{1} / 2\right)^{\prime}}\left(S_{l}\left(Q_{j}\right)\right)}\right)^{1 / 2}
\end{aligned}
$$

Where, in the last inequality, we used (6). Because $t_{j}=\ell^{2}\left(Q_{j}\right)$, for $l \geq 1$, we obtain $d\left(S_{l}\left(Q_{j}\right), Q_{j}\right) \geq 2^{l-2} \ell\left(Q_{j}\right)$. Recall that $\eta=(1 / 2)\left(n / p_{1}-n / p\right)$. Subsequently, we obtain

$$
\begin{aligned}
& \left.\left.\left|\int_{\mathbb{R}^{n}} \int_{0}^{\infty}\right| \sum_{j}\left[b, T_{s}\left(\mathscr{I}-e^{-t_{j} L}\right)^{m}\right] h_{j}(x)\right|^{2} \frac{d s}{s} v(x) d x\right|^{1 / 2} \\
& \leq C \lambda \sum_{j}\left(\sum_{l=1}^{\infty} \ell\left(Q_{j}\right)^{2\left(n / p_{1}-n / p\right)} 2^{-4(m-\eta) l}\left|Q_{j}\right|^{2 / p}\|v\|_{L^{\left(p_{1} / 2\right)^{\prime}}\left(S_{l}\left(Q_{j}\right)\right)}\right)^{1 / 2} \\
& \leq C \lambda \sum_{j}\left(\sum_{l=1}^{\infty} \ell\left(Q_{j}\right)^{2\left(n / p_{1}-n / p\right)} 2^{-4(m-\eta) l}\left|Q_{j}\right|^{2 / p}\left|2^{l+1} Q_{j}\right|^{1 /\left(p_{1} / 2\right)^{\prime}}\left(\frac{1}{\left|2^{l+1} Q_{j}\right|} \int_{2^{l+1} Q_{j}}|v(y)|^{\left(p_{1} / 2\right)^{\prime}} d y\right)^{1 /\left(p_{1} / 2\right)^{\prime}}\right)^{1 / 2} \\
& \leq C \lambda \sum_{j}\left(\sum_{l=1}^{\infty} 2^{-4(m-\eta) l+l n \cdot 1 /\left(p_{1} / 2\right)^{\prime}}\left|Q_{j}\right| \underset{y \in Q_{j}}{\operatorname{essinf}} M\left(|v|^{\left(p_{1} / 2\right)^{\prime}}\right)(y)^{1 /\left(p_{1} / 2\right)^{\prime}}\right)^{1 / 2} \\
& \leq C \lambda \sum_{j}\left(\left|Q_{j}\right| \underset{y \in Q_{j}}{\operatorname{essinf}} M\left(|v|^{\left(p_{1} / 2\right)^{\prime}}\right)(y)^{1 /\left(p_{1} / 2\right)^{\prime}}\right)^{1 / 2} \leq C \lambda \sum_{j}\left|Q_{j}\right|^{1 / 2} \underset{y \in Q_{j}}{\operatorname{essinf}} M\left(|v|^{\left(p_{1} / 2\right)^{\prime}}\right)(y)^{1 / 2\left(p_{1} / 2\right)^{\prime}} .
\end{aligned}
$$

Then, using $\left|Q_{j}\right| \sim \lambda^{-p}\|f\|_{L^{p}\left(Q_{j}\right)}^{p}$, we obtain

$$
\begin{aligned}
& \left.\left.\left|\int_{\mathbb{R}^{n}} \int_{0}^{\infty}\right| \sum_{j}\left[b, T_{s}\left(\mathscr{I}-e^{-t_{j} L}\right)^{m}\right] h_{j}(x)\right|^{2} \frac{d s}{s} v(x) d x\right|^{1 / 2} \\
& \leq C \lambda^{1+(1 / 2) p}\|f\|_{L^{p}}^{-p / 2} \\
& \quad \cdot \sum_{j}\left|Q_{j}\right| \underset{y \in Q_{j}}{\operatorname{essinf}} M\left(|v|^{\left(p_{1} / 2\right)^{\prime}}\right)(y)^{1 / 2\left(p_{1} / 2\right)^{\prime}} \\
& \leq C \lambda^{1+(1 / 2) p}\|f\|_{L^{p}}^{-p / 2}
\end{aligned}
$$

$$
\begin{aligned}
& \cdot \sum_{j} \int_{Q_{j}} M\left(|v|^{\left(p_{1} / 2\right)^{\prime}}\right)(x)^{1 / 2\left(p_{1} / 2\right)^{\prime}} d x \\
& \leq C \lambda^{1+(1 / 2) p}\|f\|_{L^{p}}^{-p / 2} \\
& \cdot \int_{\bigcup_{j} Q_{j}} M\left(|v|^{\left(p_{1} / 2\right)^{\prime}}\right)(x)^{1 / 2\left(p_{1} / 2\right)^{\prime}} d x .
\end{aligned}
$$

Then, because the Hardy-Littlewood maximal function is of weak-type $(1,1)$, we use $\|v\|_{L^{2}}=1$ and Kolmogorov's lemma to obtain 


$$
\begin{aligned}
& \left.\left.\left|\int_{\mathbb{R}^{n}} \int_{0}^{\infty}\right| \sum_{j}\left[b, T_{s}\left(\mathscr{I}-e^{-t_{j} L}\right)^{m}\right] h_{j}(x)\right|^{2} \frac{d s}{s} v(x) d x\right|^{1 / 2} \\
& \leq\left. C \lambda^{1+(1 / 2) p}\|f\|_{L^{p}\left(Q_{j}\right)}^{-p / 2}\left|\bigcup_{j}\right| Q_{j}\right|^{1-1 / 2\left(p_{1} / 2\right)^{\prime}}\left\||v|^{\left(p_{1} / 2\right)^{\prime}}\right\|_{1}^{1 / 2\left(p_{1} / 2\right)^{\prime}} \\
& \leq C \lambda^{1+(1 / 2) p}\|f\|_{L^{p}}^{-p / 2}\left|\bigcup_{j} Q_{j}\right|^{1 / 2+1 / p_{1}} .
\end{aligned}
$$

Then, we plug the estimate into (31) to obtain

$$
\begin{aligned}
I I_{2} & \leq C \lambda^{p p_{1} / 2}\|f\|_{L^{p}}^{-p p_{1} / 2}\left|\bigcup_{j} Q_{j}\right|^{p_{1}\left(1 / 2+1 / p_{1}\right)} \\
& \leq C \lambda^{p p_{1} / 2}\|f\|_{L^{p}}^{-p p_{1} / 2} \\
& \cdot\left|\left\{x \in \mathbb{R}^{n}: M\left(f^{p}\right)(x)^{1 / p}>\lambda\right\}\right|^{\left(p_{1} / 2+1\right)} .
\end{aligned}
$$

Applying $M$ of the weak-type $(1,1)$, we obtain

$$
\begin{aligned}
I I_{2} & \leq C \lambda^{p p_{1} / 2}\|f\|_{L^{p}}^{-p p_{1} / 2}\|f\|_{L^{p}}^{p\left(p_{1} / 2+1\right)} \lambda^{-p\left(p_{1} / 2+1\right)} \\
& \leq C \lambda^{-p}\|f\|_{L^{p}}^{p} .
\end{aligned}
$$

We now examine $I_{3}$. Recall that

$$
\begin{aligned}
I_{3} & =\mid\{x: \\
& \left(\int_{0}^{\infty}\left|\sum_{j}\left[b, T_{s}\left(\mathscr{I}-\left(\mathscr{I}-e^{-t_{j} L}\right)^{m}\right)\right] h_{j}(x)\right|^{2} \frac{d s}{s}\right)^{1 / 2} \\
& >\lambda\} \mid
\end{aligned}
$$

Then,

$$
\begin{aligned}
\mathscr{I}-\left(\mathscr{I}-e^{-t_{j} L}\right)^{m} & =\mathscr{I}-\sum_{k=0}^{m} C_{m}^{k} e^{-k t_{j} L} \\
& =-\sum_{k=1}^{m} C_{m}^{k} e^{-k t_{j} L} .
\end{aligned}
$$

Thus, from Chebychev's inequality,

$$
\begin{aligned}
I I_{3} \leq & \frac{C}{\lambda^{2}} \sum_{k=1}^{m}\left\|\left(\int_{0}^{\infty}\left|\left[b, T_{s} \sum_{j} e^{-k t_{j} L}\right] h_{j}\right|^{2} \frac{d s}{s}\right)^{1 / 2}\right\|_{L^{2}}^{2} \\
\leq & \frac{C}{\lambda^{2}} \sum_{k=1}^{m}\left\|\left(\int_{0}^{\infty}\left|\left[b, T_{s}\right] \sum_{j} e^{-k t_{j} L} h_{j}\right|^{2} \frac{d s}{s}\right)^{1 / 2}\right\|_{L^{2}}^{2} \\
& +\frac{C}{\lambda^{2}} \sum_{k=1}^{m}\left\|\left(\int_{0}^{\infty}\left|T_{s}\left[b, \sum_{j} e^{-k t_{j} L}\right] h_{j}\right|^{2} \frac{d s}{s}\right)^{1 / 2}\right\|^{2} \\
:= & I I_{3,1}+I I_{3,2} .
\end{aligned}
$$

We fix $1 \leq k \leq m$. Then, for $I I_{3,1}$, by (8), we obtain

$$
I I_{3,1} \leq \frac{C}{\lambda^{2}} \sum_{k=1}^{m}\left\|\sum_{j} e^{-k t_{j} L} h_{j}\right\|_{L^{2}}^{2} .
$$

Hence, by (see [9])

$$
\left\|\sum_{j} e^{-k t_{j} L} h_{j}\right\|_{L^{2}}^{2} \leq C \lambda^{2-p}\|f\|_{L^{p}}^{p},
$$

we obtain

$$
I I_{3,1} \leq C \frac{\|f\|_{L^{p}}^{p}}{\lambda^{p}} .
$$

For $I I_{3,2}$, by (7) and $\left\|L^{\alpha / 2} f\right\|_{L^{2}} \sim\left\|D^{\alpha} f\right\|_{L^{2}}$ (see [3]), we obtain

$$
\begin{gathered}
I I_{3,2} \leq \frac{C}{\lambda^{2}} \sum_{k=1}^{m}\left\|D^{\alpha} \sum_{j}\left[b, e^{-k t_{j} L}\right] h_{j}\right\|_{L^{2}}^{2} \\
\leq \frac{C}{\lambda^{2}} \sum_{k=1}^{m}\left\|L^{\alpha / 2} \sum_{j}\left[b, e^{-k t_{j} L}\right] h_{j}\right\|_{L^{2}}^{2} \\
\leq \frac{C}{\lambda^{2}}\left(\sum_{k=1}^{m}\left\|\left[b, L^{\alpha / 2}\right] \sum_{j} e^{-k t_{j} L} h_{j}\right\|_{L^{2}}^{2}\right. \\
\left.+\sum_{k=1}^{m}\left\|\sum_{j}\left[b, L^{\alpha / 2} e^{-k t_{j} L}\right] h_{j}\right\|_{L^{2}}^{2}\right) .
\end{gathered}
$$

Then,

$$
\begin{array}{r}
I I_{3,2} \leq \frac{C}{\lambda^{2}}\left(\sum_{k=1}^{m}\left\|\sum_{j} e^{-k t_{j} L} h_{j}\right\|_{L^{2}}^{2}\right. \\
\left.+\sum_{k=1}^{m}\left\|\sum_{j}\left[b, L^{\alpha / 2} e^{-k t_{j} L}\right] h_{j}\right\|_{L^{2}}^{2}\right),
\end{array}
$$


where we use the fact that $\left[b, L^{\alpha / 2}\right]$ is bounded on $L^{2}$ with the bound $\left\|(-\Delta)^{\alpha / 2} b\right\|_{B M O}$ (see [16]). Next, we estimate the abovementioned two norms, $\left\|\sum_{j} e^{-k t_{j} L} h_{j}\right\|_{L^{2}}$ and $\left\|\sum_{j}\left[b, L^{\alpha / 2} e^{-k t_{j} L}\right] h_{j}\right\|_{L^{2}}$. Now, taking $v \in L^{2}\left(\mathbb{R}^{n}\right)$ with $\|v\|_{L^{2}}=$ 1 ,

$$
\begin{aligned}
& \left|\int_{\mathbb{R}^{n}} \sum_{j} e^{-k t_{j} L} h_{j}(x) \overline{v(x)} d x\right| \\
& =\left|\sum_{j} \sum_{l=0}^{\infty} \int_{S_{l}\left(Q_{j}\right)} e^{-k t_{j} L} h_{j}(x) \overline{v(x)} d x\right| \\
& \leq \sum_{j} \sum_{l=0}^{\infty}\left(\int_{S_{l}\left(Q_{j}\right)}\left|e^{-k t_{j} L} h_{j}(x)\right|^{2} d x\right)^{1 / 2} \\
& \cdot\left(\int_{S_{l}\left(Q_{j}\right)}|v(x)|^{2} d x\right)^{1 / 2} .
\end{aligned}
$$

Note that, for all $p_{L}<r \leq 2, e^{-k t_{j} L}$ satisfies the $L^{r}-L^{2}$ offdiagonal estimates (see Lemma 3(ii)); let $t_{j}=\ell\left(Q_{j}\right)^{2}$, and we obtain (47), which is controlled by

$$
\begin{aligned}
\sum_{j} \sum_{l=0}^{\infty} e^{-4^{l}} t_{j}^{(1 / 2)(n / 2-n / r)}\left\|h_{j}\right\|_{L^{r}\left(Q_{j}\right)}\left|2^{l+1} Q_{j}\right|^{1 / 2} & \cdot\left(\frac{1}{\left|2^{l+1} Q_{j}\right|} \int_{S_{l}\left(Q_{j}\right)}|v(x)|^{2} d x\right)^{1 / 2} \\
\leq & C \lambda \sum_{j} \sum_{l=0}^{\infty} e^{-4^{l}} \ell\left(Q_{j}\right)^{(n / 2-n / r)}\left|Q_{j}\right|^{1 / r}\left|2^{l+1} Q_{j}\right|^{1 / 2} \\
& \cdot\left(\frac{1}{\left|2^{l+1} Q_{j}\right|} \int_{S_{l}\left(Q_{j}\right)}|v(x)|^{2} d x\right)^{1 / 2} \\
\leq & C \lambda \sum_{j} \sum_{l=0}^{\infty} e^{-4^{l}} 2^{l n}\left|Q_{j}\right| \\
& \cdot\left(\frac{1}{\left|2^{l+1} Q_{j}\right|} \int_{2^{l+1} Q_{j}}|v(x)|^{2} d x\right)^{1 / 2} \\
\leq & C \lambda \sum_{j}\left|Q_{j}\right| \operatorname{ess}_{y \in Q_{j}} M\left(|v|^{2}\right)^{1 / 2}(y) \\
\leq & C \lambda \int_{\cup_{j} Q_{j}} M\left(|v|^{2}\right)^{1 / 2}(x) d x . \\
\leq & \left.C \lambda\left|\bigcup_{j}\right| Q_{j}\right|^{1 / 2}\left\||v|^{2}\right\|_{L^{1}}^{1 / 2} \\
\leq & C \lambda\left|\left\{x \in \mathbb{R}^{n}: M\left(f^{p}\right)(x)^{1 / p}>\lambda\right\}\right|^{1 / 2}
\end{aligned}
$$

Thus, from (47), (48), and the fact that $M$ is of weak-type $(1,1)$, we obtain

$$
\left\|\sum_{j} e^{-k t_{j} L} h_{j}\right\|_{L^{2}} \leq C \lambda\left(\frac{\|f\|_{L^{p}}}{\lambda}\right)^{p / 2} .
$$

For the second term of $I I_{3,2},\left\|\sum_{j}\left[b, L^{\alpha / 2} e^{-k t_{j} L}\right] h_{j}\right\|_{L^{2}}$, by Lemma 6 and the same procedures performed previously (48):

$$
\begin{aligned}
& \left|\int_{\mathbb{R}^{n}} \sum_{j}\left[b, L^{\alpha / 2} e^{-k t_{j} L}\right] h_{j}(x) \overline{v(x)} d x\right| \\
& \quad=\left|\sum_{j} \sum_{l=0}^{\infty} \int_{S_{l}\left(Q_{j}\right)}\left[b, L^{\alpha / 2} e^{-k t_{j} L}\right] h_{j}(x) \overline{v(x)} d x\right| \\
& \leq \sum_{j} \sum_{l=0}^{\infty}\left(\int_{S_{l}\left(Q_{j}\right)}\left|t_{j}^{-\alpha / 2}\left[b, t_{j}^{\alpha / 2} L^{\alpha / 2} e^{-k t_{j} L}\right] h_{j}(x)\right|^{2} d x\right)^{1 / 2} \\
& \cdot\left(\int_{S_{l}\left(Q_{j}\right)}|v(x)|^{2} d x\right)^{1 / 2} \\
& \leq C \sum_{j} \sum_{l=0}^{\infty} e^{-u^{l}} t_{j}^{(1 / 2)(n / 2-n / r)}\left\|h_{j}\right\|_{L^{r}}\left|2^{l+1} Q_{j}\right|^{1 / 2}\left(\frac{1}{\left|2^{l+1} Q_{j}\right|}\right. \\
& \left.\quad \cdot \int_{S_{l}\left(Q_{j}\right)}|v(x)|^{2} d x\right)^{1 / 2} \\
& \leq C \lambda\left|\left\{x \in \mathbb{R}^{n}: M\left(f^{p}\right)(x)^{1 / p}>\lambda\right\}\right|^{1 / 2} .
\end{aligned}
$$

Then, we have

$$
\left\|\sum_{j}\left[b, L^{\alpha / 2} e^{-k t_{j} L}\right] h_{j}\right\|_{L^{2}} \leq C \lambda\left(\frac{\|f\|_{L^{p}}}{\lambda}\right)^{p / 2} .
$$

Combining (49) and (51), we obtain

$$
I I_{3,2} \leq C \lambda^{-p}\|f\|_{L^{p}}^{p} .
$$

Combining the estimates of $I I_{3,1}$ and $I I_{3,2}$, we obtain

$$
I I_{3} \leq C \frac{\|f\|_{L^{p}}^{p}}{\lambda^{p}} .
$$

The proof of Theorem 1 is now completed by combining the estimates of $I, I I_{1}$, and $I I_{2}$ with $I I_{3}$.

\section{Proof of Theorem 2}

First, we introduce a lemma that will be used to prove Theorem 2.

Lemma 8 (see [16]). For $0<\alpha<1$, let $b \in I_{\alpha}(B M O)$ ). Suppose that $\left\{\theta_{s}\right\}_{s>0}$ is a family of operators satisfying

$$
\begin{aligned}
& \left\|\theta_{s}\left(f 1_{S_{k}(Q)}\right)\right\|_{L^{2}(Q)}^{2} \\
& \quad \leq C 2^{-n k}\left(\frac{\sqrt{s}}{2^{k} \ell(Q)}\right)^{2 m+2}\|f\|_{L^{2}\left(S_{k}(Q)\right)}^{2}
\end{aligned}
$$


for an $m \geq 1$ and all $0<s \leq C \ell(Q)$. Assume that

$$
\begin{aligned}
\sup _{s>0}\left\|\theta_{s}\right\|_{L^{2} \rightarrow L^{2}} & \leq C, \\
\theta_{s} 1 & =0
\end{aligned}
$$

in the sense of $L_{l o c}^{2}$ for all $s>0$. Then, we have

$$
\int_{\mathbb{R}^{n}} \int_{0}^{\infty}\left|s^{-\alpha / 2} \theta_{s} f(x)\right|^{2} \frac{d x d s}{s} \leq C\left\|D^{\alpha} f\right\|_{L^{2}}^{2} .
$$

If, in addition,

$$
\left\|\theta_{s} \operatorname{div}\right\|_{L^{2} \rightarrow L^{2}} \leq \frac{C}{\sqrt{s}}
$$

then

$$
\begin{aligned}
& \int_{\mathbb{R}^{n}} \int_{0}^{\infty}\left|\left[b, s^{-\alpha / 2} \theta_{s}\right] f(x)\right|^{2} \frac{d x d s}{s} \\
& \leq C\left\|D^{\alpha} b\right\|_{B M O}^{2}\|f\|_{L^{2}}^{2} .
\end{aligned}
$$

Recall that

$$
G_{L ; b}^{\alpha} f(x)=\left(\int_{0}^{\infty}\left|\left[b, s^{(1-\alpha) / 2} \nabla e^{-s L}\right] f(x)\right|^{2} \frac{d s}{s}\right)^{1 / 2} .
$$

The proof of Theorem 2 is presented in two steps.

Step 1 (the $L^{2}$ boundedness). Let $\theta_{s}=s^{1 / 2} \nabla e^{-s L}$. By Lemma 8, we only need to prove that $s^{1 / 2} \nabla e^{-s L}$ satisfies (54)-(56) and (58). Because $\left\|s^{1 / 2} \nabla e^{-s L} \operatorname{div}\right\|_{L^{2} \longrightarrow L^{2}} \leq C / s$, it is easy to verify that $s^{1 / 2} \nabla e^{-s L}$ satisfies (54), (56), and (58). Moreover, it is easy to verify that $s^{1 / 2} \nabla e^{-s L}$ satisfies (55) by the $L^{2}$ off-diagonal estimate of the operator $s^{1 / 2} \nabla e^{-s L}$ (see Lemma 3(i, iii)). We obtain

$$
\begin{aligned}
\left\|G_{L}^{\alpha} f(x)\right\|_{L^{2}} & \leq C\left\|D^{\alpha} f\right\|_{L^{2}}, \\
\left\|G_{L ; b}^{\alpha} f(x)\right\|_{L^{2}} & \leq C\left\|D^{\alpha} b\right\|_{B M O}\|f\|_{L^{2}} .
\end{aligned}
$$

Step 2 (weak-type $(p, p)$ boundedness for $p_{L}<p<2$ ). We first prove that the commutator is of the weak-type $(p, p)$ for $p_{L}<p<2$. We apply Theorem 1 with $T_{s}=s^{(1-\alpha) / 2} \nabla e^{-s L}$ to prove this result. Because $G_{L}^{\alpha}$ and $G_{L ; b}^{\alpha}$ are bounded on $L^{2}\left(\mathbb{R}^{n}\right)$, verification of $\left\{s^{(1-\alpha) / 2} \nabla e^{-s L}\right\}_{s>0}$ satisfies (6). Taking $p<2<$ $p_{1}$, by the Minkowski inequality, we obtain

$$
\begin{aligned}
& \left\|\left(\int_{0}^{\infty}\left|\left[b, s^{1 / 2} \nabla e^{-s L}\left(\mathscr{I}-e^{-t L}\right)^{m}\right] f\right|^{2} \frac{d s}{s^{1+\alpha}}\right)^{1 / 2}\right\|_{L^{p_{1}}(F)} \\
& \leq\left(\int_{0}^{\infty}\left\|\left[b, s^{1 / 2} \nabla e^{-s L}\left(\mathscr{I}-e^{-t L}\right)^{m}\right] f\right\|_{L^{p_{1}}(F)}^{2} \frac{d s}{s^{1+\alpha}}\right)^{1 / 2} \\
& =C\left(\int_{0}^{t} \cdots+\int_{t}^{\infty} \cdots\right)^{1 / 2}=C\left(I_{t}+I I_{t}\right)^{1 / 2} .
\end{aligned}
$$

Now, we study each operator separately. For the first operator, we have

$$
\left(\mathscr{I}-e^{-t L}\right)^{m}=\sum_{k=0}^{m} C_{m}^{k}(-1)^{k} e^{-k t L}=\mathscr{I}+\sum_{k=1}^{m} c_{k} e^{-k t L},
$$

and, then,

$$
\begin{aligned}
I_{t} \leq & \int_{0}^{t}\left\|\left[b, s^{1 / 2} \nabla e^{-s L}\right] f\right\|_{L^{p_{1}}(F)}^{2} \frac{d s}{s^{1+\alpha}} \\
& +\sum_{k=1}^{m} c_{k} \int_{0}^{t}\left\|\left[b, s^{1 / 2} \nabla e^{-s L} e^{-k t L}\right] f\right\|_{L^{p_{1}}(F)}^{2} \frac{d s}{s^{1+\alpha}} \\
= & I_{t, 0}+\sum_{k=1}^{m} c_{k} I_{t, k} .
\end{aligned}
$$

For $I_{t, 0}$, by Lemma 7(i), we have

$$
\begin{aligned}
& I_{t, 0} \leq \int_{0}^{t}\left\|\left[b, s \frac{1-\alpha}{2} \nabla e^{-s L}\right] f\right\|_{L^{p_{1}(F)}}^{2} \frac{d s}{s} \\
& \leq C\|b\|_{\operatorname{Lip}_{\alpha}}^{2}\|f\|_{L^{p}(E)}^{2} \int_{0}^{t} e^{-d(E, F)^{2} / c s} s^{\left(n / p_{1}-n / p\right)} \frac{d s}{s} \\
& =C\|b\|_{\operatorname{Lip}_{\alpha}}^{2}\|f\|_{L^{p}(E)}^{2} \int_{0}^{t} e^{-d(E, F)^{2} / c s} s^{2 \eta} \frac{d s}{s} \\
& =C\|b\|_{\operatorname{Lip}_{\alpha}}^{2}\|f\|_{L^{p}(E)}^{2} t^{2 \eta} \int_{1}^{\infty} e^{-\left(d(E, F)^{2} / c t\right) s} s^{-2 \eta} \frac{d s}{s} \\
& \leq C\|b\|_{\operatorname{Lip}_{\alpha}}^{2}\|f\|_{L^{p}(E)}^{2} \\
& \cdot t^{2 \eta} \int_{1}^{\infty}\left(\frac{d(E, F)^{2}}{c t} s\right)^{-2(m-\eta)} s^{-2 \eta} \frac{d s}{s} \\
& \leq C\|b\|_{\operatorname{Lip}_{\alpha}}^{2}\|f\|_{L^{p}(E)}^{2} t^{2 \eta}\left(\frac{d(E, F)^{2}}{t}\right)^{-2(m-\eta)},
\end{aligned}
$$

where $\eta=(1 / 2)\left(n / p_{1}-n / p\right)$. Now, with $1 \leq k \leq m$, by the commutative property of the semigroup and Lemma 7(i), we obtain

$$
\begin{aligned}
I_{t, k} \leq & \int_{0}^{t}\left\|\left[b,(s+k t)^{(1-\alpha) / 2} \nabla e^{-(s+k t) L}\right] f\right\|_{L^{p_{1}}(F)}^{2} \\
& \cdot s(s+k t)^{\alpha-1} \frac{d s}{s^{1+\alpha}} \\
\leq & C\|b\|_{\operatorname{Lip}_{\alpha}}^{2}\|f\|_{L^{p}(E)}^{2} \\
& \cdot \int_{0}^{t} e^{-d(E, F)^{2} / c(s+k t)}(s+k t)^{\left(n / p_{1}-n / p\right)}(s+k t)^{\alpha-1} s \frac{d s}{s^{1+\alpha}} \\
\leq & C\|b\|_{\operatorname{Lip}_{\alpha}}^{2}\|f\|_{L^{p}(E)}^{2} e^{-d(E, F)^{2} / c t} t^{\left(n / p_{1}-n / p+\alpha-1\right)} \int_{0}^{t} s^{-\alpha} d s \\
\leq & C\|b\|_{\operatorname{Lip}_{\alpha}}^{2}\|f\|_{L^{p}(E)}^{2} e^{-d(E, F)^{2} / c t} t^{\left(n / p_{1}-n / p\right)} \\
\leq & C\|b\|_{\operatorname{Lip}_{\alpha}}^{2}\|f\|_{L^{p}(E)}^{2}\left(\frac{d(E, F)^{2}}{t}\right)^{-2(m-\eta)} t^{2 \eta} .
\end{aligned}
$$


Collecting this estimate and the one proved for $I_{t, 0}$, we obtain

$$
\begin{aligned}
I_{t} & \leq I_{t, 0}+\sum_{k=1}^{m}\left|c_{k}\right| I_{t, k} \\
& \leq C\|b\|_{\operatorname{Lip}_{\alpha}}^{2} t^{2 \eta}\left(\frac{d(E, F)^{2}}{t}\right)^{-2(m-\eta)}\|f\|_{L^{p}(E)}^{2} .
\end{aligned}
$$

Next, we proceed with the estimate of $I I_{t}$ :

$$
\begin{aligned}
I I_{t} & =\int_{t}^{\infty}\left\|\left[b, s^{1 / 2} \nabla e^{-s L}\left(\mathscr{I}-e^{-t L}\right)^{m}\right] f\right\|_{L^{p_{1}}(F)}^{2} \frac{d s}{s^{1+\alpha}} \\
& =C \int_{t}^{\infty} \|\left[b, s^{1 / 2} \nabla e^{-(m+1) s L}\left(\mathscr{I}-e^{-t L}\right)^{m}\right] \\
& \cdot f \|_{L^{p_{1}}(F)}^{2} \frac{d s}{s^{1+\alpha}} \\
& =C \int_{t}^{\infty} \|\left[b, s^{1 / 2} \nabla e^{-s L}\left(e^{-s L}-e^{-(s+t) L}\right)^{m}\right] \\
& \cdot f \|_{L^{p_{1}}(F)}^{2} \frac{d s}{s^{1+\alpha}} .
\end{aligned}
$$

Let $E, F$ be two closed sets and $g$ be such that supp $g \subset E$. For $t \leq s$, by a similar previous argument [9], we can prove that

$$
\begin{aligned}
& \left\|\frac{s}{t}\left(e^{-s L}-e^{(s+t) L}\right) g\right\|_{L^{p_{1}}(F)} \\
& \quad \leq C s^{(1 / 2)\left(n / p_{1}-n / p_{0}\right)} e^{-d(E, F)^{2} / c s}\|g\|_{L^{p}(E)}
\end{aligned}
$$

uniformly on $t$. Then, by Lemma 4 , we have

$$
\begin{aligned}
& \left\|\left(e^{-s L}-e^{(s+t) L}\right)^{m} g\right\|_{L^{p_{1}}(F)} \\
& \quad \leq C\left(\frac{t}{s}\right)^{m} s^{(1 / 2)\left(n / p_{1}-n / p_{0}\right)} e^{-d(E, F)^{2} / c s}\|g\|_{L^{p}(E)} .
\end{aligned}
$$

We write $I I_{t}$ as follows:

$$
\begin{aligned}
I I_{t} & \leq C \int_{t}^{\infty} \|\left[b, s^{1 / 2} \nabla e^{-s L}\right]\left(e^{-s L}-e^{-(s+t) L}\right)^{m} \\
& \cdot f \|_{L^{p_{1}(F)}}^{2} \frac{d s}{s^{1+\alpha}} \\
& +C \int_{t}^{\infty} \| s^{1 / 2} \nabla e^{-s L}\left[b,\left(e^{-s L}-e^{-(s+t) L}\right)^{m}\right] \\
& \cdot f \|_{L^{p_{1}(F)}}^{2} \frac{d s}{s^{1+\alpha}}:=G_{1}+G_{2} .
\end{aligned}
$$

First, we consider $G_{1}$ :

$$
\begin{aligned}
G_{1} & =\int_{t}^{\infty} \|\left(\left[b, s^{(1-\alpha) / 2} \nabla e^{-s L}\right]\right) \\
& \circ\left(e^{-s L}-e^{-(s+t) L}\right)^{m} f \|_{L^{p_{1}}(F)}^{2} \frac{d s}{s} .
\end{aligned}
$$

Let us observe that, because of (71) and Lemma 7(i) in the composition of the operators, each of them verifies an offdiagonal estimate. This fact allows us to employ Lemma 4 to obtain

$$
\begin{aligned}
G_{1} \leq C\|b\|_{\operatorname{Lip}_{\alpha}}^{2}\|f\|_{L^{p}(E)}^{2} & \cdot \int_{t}^{\infty} e^{-d(E, F)^{2} / c s}\left(\frac{t}{s}\right)^{2 m} s^{\left(n / p_{1}-n / p\right)} \frac{d s}{s} \\
= & C\|b\|_{\operatorname{Lip}_{\alpha}}^{2}\|f\|_{L^{p}(E)}^{2} \int_{t}^{\infty} e^{-d(E, F)^{2} / c s}\left(\frac{t}{s}\right)^{2 m} s^{2 \eta} \frac{d s}{s} \\
= & C\|b\|_{\operatorname{Lip}_{\alpha}}^{2}\|f\|_{L^{p}(E)}^{2} t^{2 \eta} \int_{0}^{1} e^{-\left(d(E, F)^{2} / c t\right) s} s^{2 m-2 \eta} \frac{d s}{s} \\
\leq & C\|b\|_{\operatorname{Lip}_{\alpha}}^{2}\|f\|_{L^{p}(E)}^{2} t^{2 \eta} \int_{0}^{\infty} e^{-\left(d(E, F)^{2} / c t\right) s} s^{2 m-2 \eta} \frac{d s}{s} \\
= & C\|b\|_{\operatorname{Lip}_{\alpha}}^{2}\|f\|_{L^{p}(E)}^{2} t^{2 \eta}\left(\frac{d(E, F)^{2}}{t}\right)^{-2(m-\eta)} \\
& \cdot \int_{0}^{\infty} e^{-s} s^{2(m-\eta)} \frac{d s}{s} \\
\leq & C\|b\|_{\operatorname{Lip}_{\alpha}}^{2}\|f\|_{L^{p}(E)}^{2} t^{2 \eta}\left(\frac{d(E, F)^{2}}{t}\right)^{-2(m-\eta)},
\end{aligned}
$$

where $\eta=(1 / 2)\left(n / p_{1}-n / p\right)$. From Lemma 5 and (71), it is easy to obtain

$$
\begin{aligned}
& \left\|\left[b, s^{-\alpha / 2}\left(e^{-s L}-e^{(s+t) L}\right)^{m}\right] g\right\|_{L^{p_{1}}(F)} \\
& \quad \leq C\|b\|_{\operatorname{Lip}_{\alpha}}\left(\frac{t}{s}\right)^{m} s^{(1 / 2)\left(n / p_{1}-n / p_{0}\right)} e^{-d(E, F)^{2} / c s}\|g\|_{L^{p}(E)} .
\end{aligned}
$$

Finally, for $G_{2}$,

$$
\begin{aligned}
G_{2} & =\int_{t}^{\infty} \|\left(s^{1 / 2} \nabla e^{-s L}\right) \\
\circ & {\left[b, s^{-\alpha / 2}\left(e^{-s L}-e^{-(s+t) L}\right)^{m}\right] f \|_{L^{p_{1}}(F)}^{2} \frac{d s}{s} . }
\end{aligned}
$$

We know that the composition of the operators above (75) or Lemma 3 verifies an off-diagonal estimate. It follows from Lemma 4 that

$$
\begin{aligned}
G_{2} & \leq C\|b\|_{\operatorname{Lip}_{\alpha}}^{2}\|f\|_{L^{p}(E)}^{2} \int_{t}^{\infty} e^{-d(E, F)^{2} / c s}\left(\frac{t}{s}\right)^{2 m} s^{2 \eta} \frac{d s}{s} \\
& \leq C\|b\|_{\operatorname{Lip}_{\alpha}}^{2}\|f\|_{L^{p}(E)}^{2} t^{2 \eta}\left(\frac{d(E, F)^{2}}{t}\right)^{-2(m-\eta)} .
\end{aligned}
$$

Collecting the estimates for $G_{1}$ and $G_{2}$, we obtain

$$
I I_{t} \leq C\|b\|_{\operatorname{Lip}_{\alpha}}^{2} t^{2 \eta}\left(\frac{d(E, F)^{2}}{t}\right)^{-2(m-\eta)}\|f\|_{L^{p}(E)}^{2},
$$

which combined with the estimate of $I_{t}$ verify that $\left\{s^{(1-\alpha) / 2} \nabla e^{-s L}\right\}_{s>0}$ satisfies (6). Thus, we have proven Theorem 2 . 


\section{Data Availability}

No data were used to support this study.

\section{Conflicts of Interest}

The authors declare that they have no conflicts of interest.

\section{Acknowledgments}

Y. Chen is supported by NSFC (11471033) and the Fundamental Research Funds for the Central Universities (FRF-BR-17001B).

\section{References}

[1] R. S. Strichartz, "Bounded mean oscillation and Sobolev spaces," Indiana University Mathematics Journal, vol. 29, no. 4, pp. 539-558, 1980.

[2] M. E. Taylor, "Commutator estimates for Hölder continuous and bmo-Sobolev multipliers," Proceedings of the American Mathematical Society, vol. 143, no. 12, pp. 5265-5274, 2015.

[3] P. Auscher, "On necessary and sufficient conditions for $L^{p_{-}}$ estimates of Riesz transforms associated to elliptic operators on $\mathbb{R}^{n}$ and related estimates," Memoirs of the American Mathematical Society, vol. 186, no. 871, 2007.

[4] P. Auscher and J. M. Martell, "Weighted norm inequalities, offdiagonal estimates and elliptic operators. Part III: Harmonic analysis of elliptic operators," Journal of Functional Analysis, vol. 241, no. 2, pp. 703-746, 2006.

[5] P. Auscher and J. M. Martell, "Weighted norm inequalities, offdiagonal estimates and elliptic operators. Part II: Off-diagonal estimates on spaces of homogeneous types," Journal of Evolution Equations, vol. 7, no. 2, pp. 265-316, 2007.

[6] Y. Chen and Y. Ding, " $L^{2}$ boundedness for commutator of rough singular integral with variable kernel," Revista Matematica Iberoamericana, vol. 24, no. 2, pp. 531-547, 2008.

[7] X. T. Duong and A. McIntosh, "Functional calculi of secondorder elliptic partial differential operators with bounded measurable coefficients," The Journal of Geometric Analysis, vol. 6, no. 2, pp. 181-205, 1996.

[8] X. T. Duong and L. Yan, "Duality of Hardy and BMO spaces associated with operators with heat kernel bounds," Journal of the American Mathematical Society, vol. 18, no. 4, pp. 943-973, 2005.

[9] S. Hofmann and J. M. Martell, " $L^{p}$ bounds for Riesz transforms and square roots associated to second order elliptic operators," Publicacions Matematiques, vol. 47, no. 2, pp. 497-515, 2003.

[10] Q. Chen and Z. Zhang, "Boundedness of a class of super singular integral operators and the associated commutators," Science China Mathematics, vol. 47, no. 6, pp. 842-853, 2004.

[11] Y. Chen, Y. Ding, and G. Hong, "Commutators with fractional differentiation and new characterizations of BMO-Sobolev spaces," Analysis \& PDE, vol. 9, pp. 1497-1522, 2016.

[12] Y. Guo, M. Wang, and Y. Tang, "Higher regularity of global attractors of a weakly dissipative fractional Korteweg de Vries equation," Journal of Mathematical Physics, vol. 56, no. 12, 2015.

[13] J. L. Lewis and M. A. Murray, "Regularity properties of commutators and layer potentials associated to the heat equation," Transactions of the American Mathematical Society, vol. 328, no. 2, pp. 815-842, 1991.
[14] M. A. Murray, "Commutators with fractional differentiation and BMO Sobolev spaces," Indiana University Mathematics Journal, vol. 34, no. 1, pp. 205-215, 1985.

[15] Y. P. Chen, Y. Ding, and S. Hofmann, "The commutator of the Kato square root for second order elliptic operators on $\mathbb{R}^{n}$," Acta Mathematica Sinica, vol. 32, no. 10, pp. 1121-1144, 2016.

[16] Y. Chen, Q. Deng, and Y. Ding, "Commutators with Fractional Differentiation for Second Order Elliptic Operators on $\mathbb{R}^{n \text { ". }}$ 


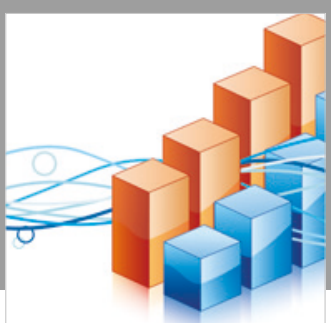

Advances in

Operations Research

\section{-n-m}
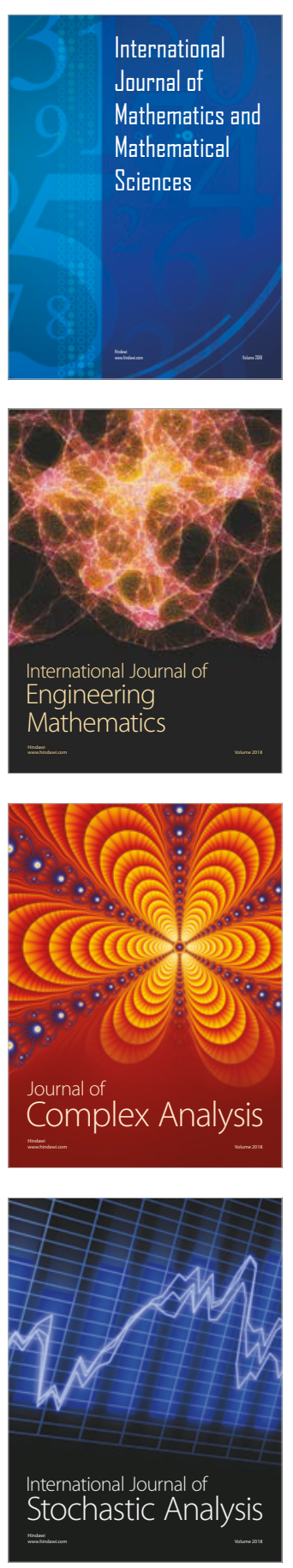
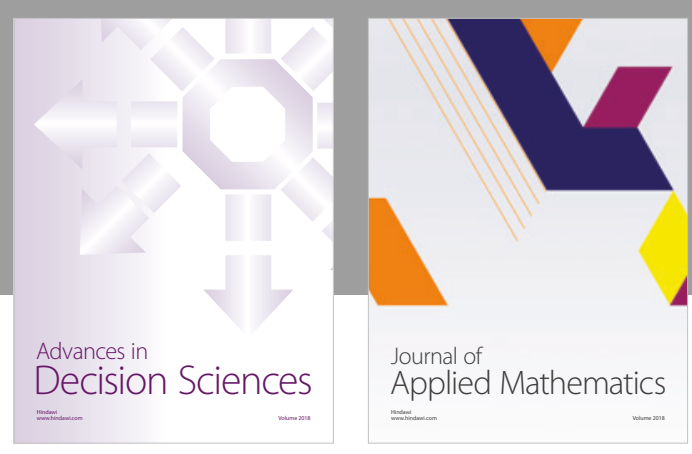

Journal of

Applied Mathematics
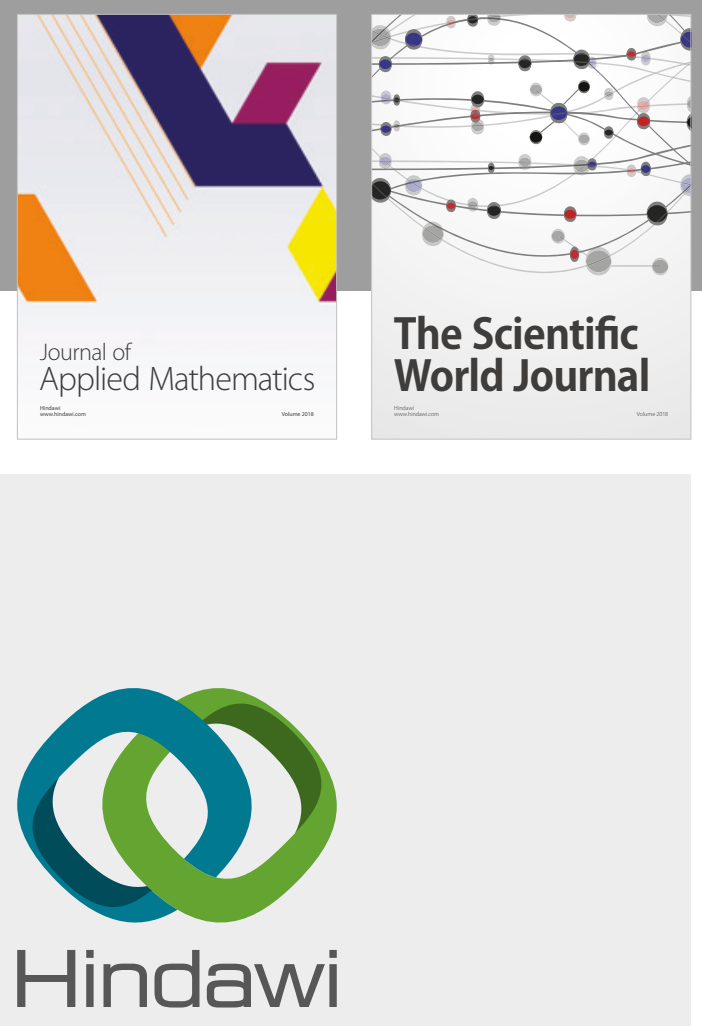

Submit your manuscripts at

www.hindawi.com

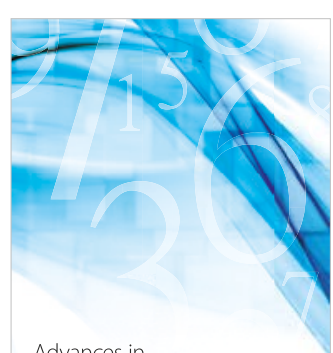

Advances in
Numerical Analysis
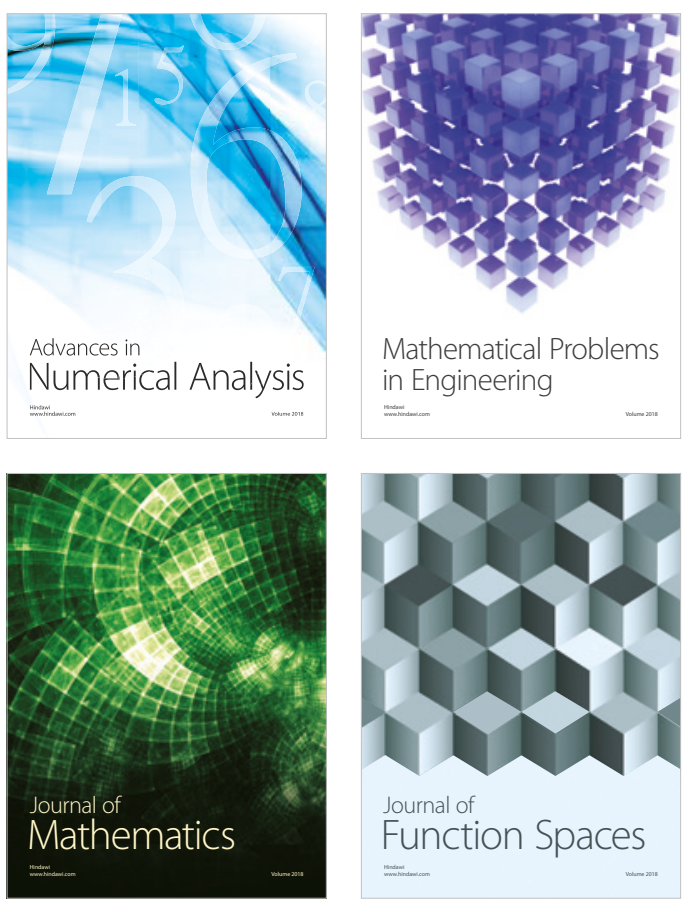

Mathematical Problems in Engineering

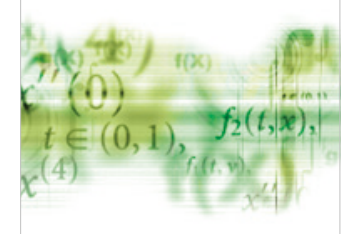

International Journal of

Differential Equations

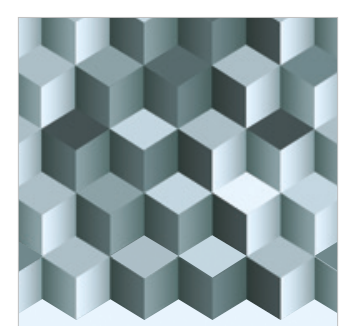

Journal of

Function Spaces

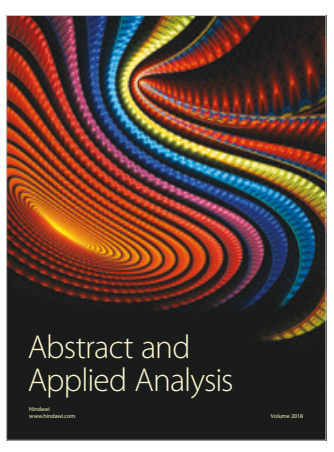

The Scientific

World Journal

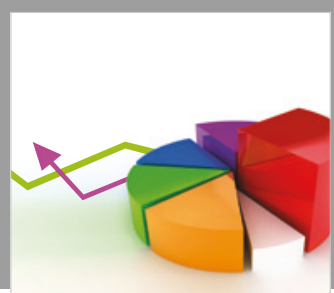

Journal of

Probability and Statistics
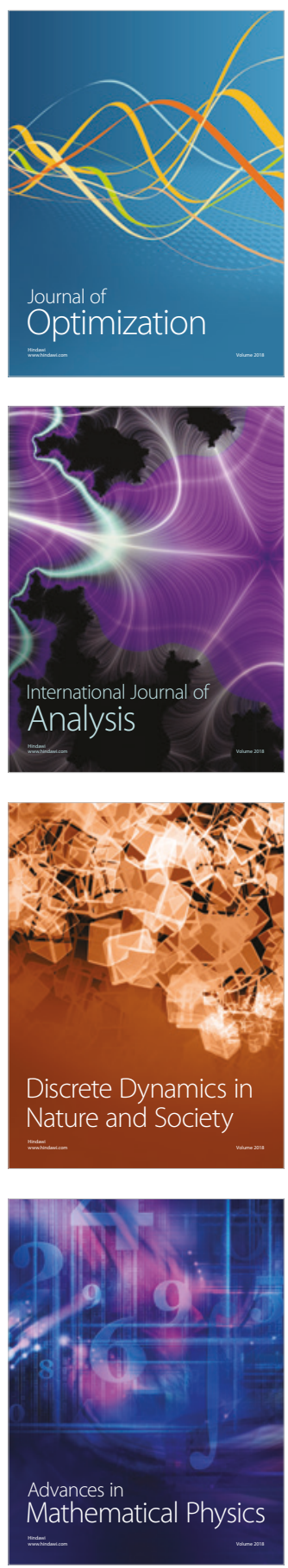